\title{
Study on Tool Condition Parameters Intended for Smart Tool Management in Filleted End Milling
}

\author{
Tsutomu Sekine ${ }^{1}$ \\ 1 Department of Systems Design Engineering, Faculty of Science and Technology, Seikei University, Tokyo, Japan \\ * Corresponding author's email: ts_s@outlook.com
}

\begin{abstract}
Filleted end milling is commonly used as a versatile manufacturing process, whereas the optimization for tool condition management is one of the continuously noticed topics. It can also contribute to the achievement of Sustainable Development Goals. A lot of outcomes have been reported so far in the experimental investigations of tool condition parameters. However, the findings and knowledge from theoretical perspective are relatively scarce to advance smart tool management. Hence, the aim of this study is to give theoretical consideration with tool condition parameters. The investigations focus on filleted end milling with some variations of machining conditions. Several tool condition parameters were theoretically proposed after illustrating geometrical modeling with variable description. Then, the demonstrations and discussion were made based on the computational results in filleted end milling. The results visually and numerically ascertained novel findings regarding several characteristics of tool condition parameters.
\end{abstract}

Keywords: tool condition parameter, machining condition, optimization, multi-axis $\mathrm{CNC}$ machining, filleted end mill.

\section{INTRODUCTION}

These days, Sustainable Development Goals have gradually come to pervade various aspects of our society. Sustainable manufacturing has resultantly been attracting global-scale attention from industries, academics, governments, etc. over the last decades $[1,2]$. Under the backgrounds, the contributions with high potential were made from a variety of perspectives [3-6]. In mechanical machining, sustainability appearing in each stage of the process is recognizable as one of the important, challenging opportunities toward the achievement of Sustainable Development Goals [7]. Moreover, it is well known that poor cutting tool management leads to a tremendous loss of productivity and profits. There are various viewpoints of studies focusing on efficient tool management not only to enlarge tool life but also to prevent tool wear and failure [8-10]. Tool condition monitoring has been conducted as one of the potential methods in some of these studies [11-14]. The smart approach is also being proposed with information and communication technologies $[15,16]$. The sophistication of these methods is desired more and more for enhancing machining efficiency, reducing the frequency of tool replacement and so on. Although experimental investigation of the controlling factors has been made in direct and indirect manners, the theoretical one is still immature to implement it into smart manufacturing system. In addition, it is computationally riskful that the extant factors include ambiguity. Thus, the further essential factors must have great potential to establish the smart tool management exploiting one of the sustainable breakthroughs in manufacturing process. They can also inspire the development of suitable hybrid approaches with theory and practice.

Milling is one of the core technologies with a versatile, highly-automated process in today's industry. Computer numerical controlled (CNC) machine tools have broadly used to advance manufacturing automation [17-19]. CNC machining 
technology gradually pervaded its applicable fields with the development of CNC machine tools. Computer-aided technologies have also added impetus to the technological advancement. Computer-aided design became one of the driving forces to create attractive products [20]. A designed shape made in computer-aided design has indispensable influence on product manufacturing. Computer-aided manufacturing is also a crucial technology for the modern production $[21,22]$. A process plan with machining parameters' selection in computer-aided manufacturing is vital for surface quality, machining accuracy, etc. in practical manufacturing [23-30]. These technologies are playing an essential role in smart manufacturing, whereas the accumulated findings and knowledge are still insufficient for the practical use in smart technologies.

There are various methodologies aimed at an optimization of machining parameters in milling. Cutting force is a high-interest topic not only to avoid tool failure but also to enhance finish surface quality. Lazoglu et al. reported a feedrate scheduling technique based on their force model [31]. Habibi et al. also proposed a computationally-inexpensive approach minimizing flute engagement to adjust tool orientation for optimizing surface errors in five axis ball end milling [32]. Moreover, cutter/workpiece engagement is one of the important factors to figure out suitable processing parameters. Zhang et al. studied an optimization of tool orientation in 5-axis ball-end milling through a model of cutter/workpiece engagement [33]. Chip thickness is theoretically modeled to determine the other machining factors. Lotfi et al. provided a model of instantaneous undeformed chip thickness [34]. Residual stress in machined surface is also a measure to optimize processing parameters; in addition, Masmiati and Sarhan revealed an influence of cutting parameters on residual stress in machined surfaces [35]. The other related works were also published in many kinds of medium [36-39]. Although there are dominant processing parameters' optimizations from various perspectives, a few comprehensive strategies have been proposed to determine machining conditions in filleted end milling. Accordingly, the accumulation of findings and knowledge is imperative for intelligent use of information toward the development of smart technologies.

In this study, theoretical investigations with tool condition parameters were given for the further advancement of smart manufacturing. They focus particularly on filleted end milling with some variations of tool rotational speed, feed rate, and tool orientation. Several tool condition parameters will be theoretically proposed after illustrating geometrical modeling with variable description. Then, the demonstrations and discussion will be made based on the computational results in filleted end milling. The results visually and numerically will reveal novel findings regarding several characteristics of tool condition parameters.

\section{Modeling with variable description}

Several coordinate systems is firstly provided to express a machining state of filleted end milling. Henceforth, an axis of a coordinate system is invariably denoted by a normalized vector. This study introduces three coordinate systems as shown in Figure 1.

These right-handed coordinate systems are labelled as G, M, and T. G coordinate system comprises $\mathbf{X}, \mathbf{Y}, \mathbf{Z}$ axes, whose components are $(1,0,0),(0,1,0)$, and $(0,0,1)$, respectively. $\mathrm{M}$ coordinate system is also defined based on a surface to be machined. MZ is a unit surface normal on a workpiece. MY is orthogonal to MX, and it is oriented to the scanning direction of a tool; moreover, MY can be obtained from the cross product of $\mathbf{M X}$ and $\mathbf{M Z}$.

Two sections are instantaneously set to consider the other coordinate system and inclination angles in filleted end milling. The one is a section based on the scanning direction of a tool (i.e., $\mathbf{M Z}$ - MX plane), while the other is a section based on the cross-feed direction perpendicular to the scanning direction (i.e., MZ

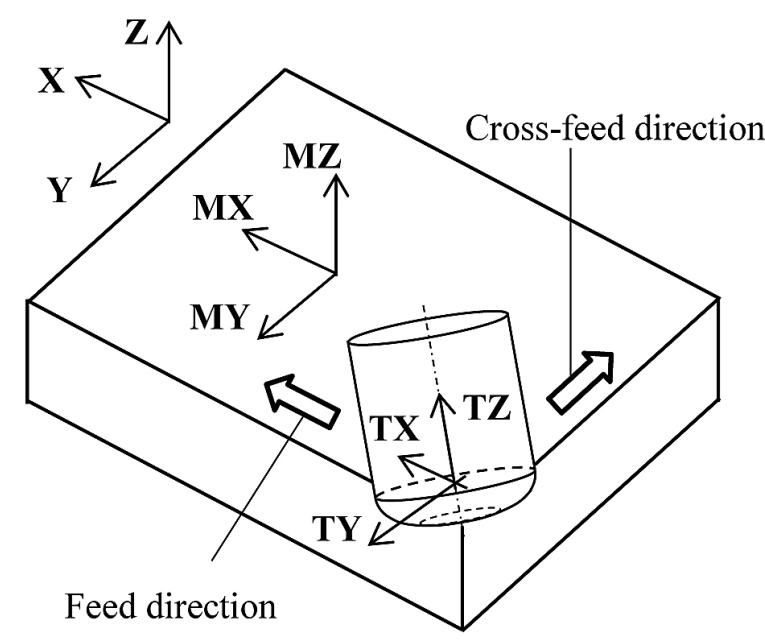

Fig. 1. Three coordinate systems in filleted end milling 


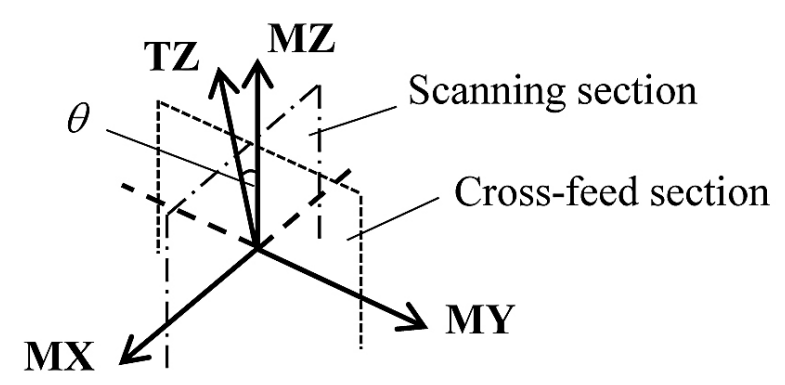

Fig. 2. Two instantaneous sections and tool inclination angle $\theta$ between $\mathbf{M Z}$ and $\mathbf{T Z}$

- MY plane). Let these planes be scanning section and cross-feed section, respectively. T coordinate system can be expressed using the two sections. The position of $\mathbf{T Z}$ corresponds to that of the rotational axis of a tool, and the direction is oriented to the shank side of tool. As illustrated in Figure 2, tool inclination angle $\theta$ denotes the angle between $\mathbf{M Z}$ and $\mathbf{T Z}$. TX is geometrically calculated as a vector perpendicular to $\mathbf{T Z}$. The direction depends on the directional relationship between TZ and MZ. Specifically, the angle between TX and MX - MY plane is inevitably set to be $\theta$. Then, $\mathbf{T Y}$ can be obtained from the cross product of $\mathbf{T X}$ and $\mathbf{T Z}$.

The angles $\theta_{S}$ and $\theta_{c}$ are additionally introduced to derive $\theta$. As shown in Figure $3, \theta_{s}$ is the inclination angle on scanning section, designating the angle between $\mathbf{M Z}$ and the orthogonal projection of $\mathbf{T Z}$ onto scanning section. Likewise, $\theta_{c}$ is the inclination angle on cross-feed section, indicating the angle between $\mathbf{M Z}$ and the orthogonal projection of $\mathbf{T Z}$ onto cross-feed section. Counterclockwise rotation is the positive rotational direction of $\theta_{s}$ and $\theta_{c}$.

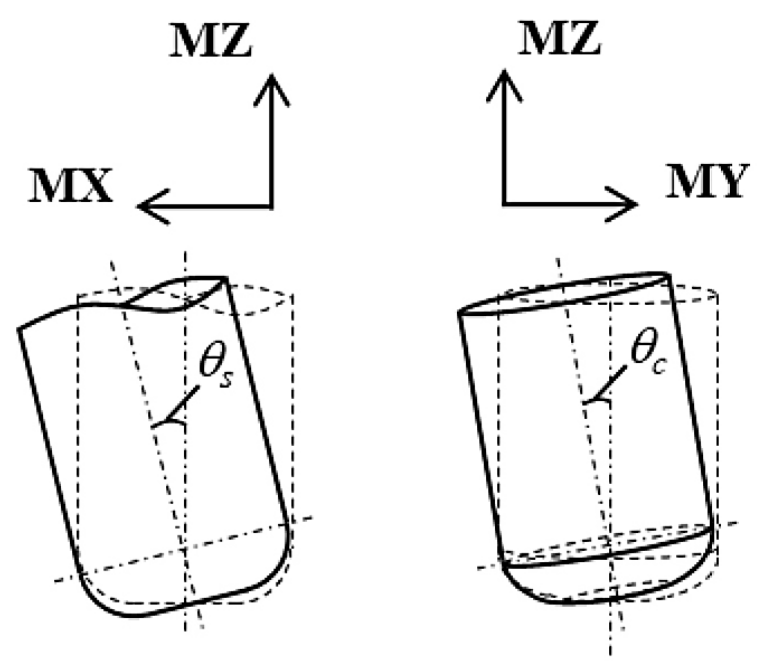

Fig. 3. Inclination angles $\theta_{S}$ and $\theta_{C}$
Given that $\mathrm{M}$ coordinate system coincides completely with $\mathrm{G}$ coordinate system for simple modeling of filleted end milling, $\theta_{S}$ and $\theta_{c}$ can be calculated as follows:

$$
\begin{gathered}
\theta_{s}=\cos ^{-1}\left(\frac{T Z_{z}}{\sqrt{T Z_{y}{ }^{2}+T Z_{z}^{2}}}\right) \\
\theta_{c}=\cos ^{-1}\left(\frac{T Z_{z}}{\sqrt{T Z_{x}^{2}+T Z_{z}^{2}}}\right)
\end{gathered}
$$

where: $T Z_{X}, T Z_{y}$, and $T Z_{Z}$ are three components of $\mathbf{T Z}$ in each axis of $\mathrm{G}$ coordinate system. Since $\mathbf{T Z}$ is given as a normalized vector,

$$
T Z_{x}^{2}+T Z_{y}^{2}+T Z_{z}^{2}=1
$$

Hence, Equations (1) and (2) can lead to the following components of TZ:

$$
\begin{gathered}
T Z_{x}=T Z_{z} \tan \theta_{s} \\
T Z_{y}=-T Z_{z} \tan \theta_{c}
\end{gathered}
$$

Substituting Equations (4) and (5) to Equation (3) provides the following formula:

$$
T Z_{z}=\sqrt{\frac{1}{\tan ^{2} \theta_{c}+\tan ^{2} \theta_{s}+1}}
$$

Accordingly, the angle $\theta$ can be offered as an inverse trigonometric function with $\mathrm{TZ}_{\mathrm{Z}}$ :

$\theta=\cos ^{-1}\left(\frac{T Z_{z}}{\sqrt{T Z_{x}^{2}+T Z_{y}^{2}+T Z_{z}^{2}}}\right)=\cos ^{-1}\left(T Z_{z}\right)(7)$

Figure 4 illustrates the positional relationship of two cutting edges in filleted end mill. Although there exist various kinds of cutting edge geometry, this study focuses on a filleted end mill with two flutes and without a helix angle of cutting edges. It means that the filleted end milling is not oblique process. With reference to Figure 4, a cutting point on a cutting edge in T coordinate system TPe can be calculated as follows: when $d_{a e} \geq R-R_{c r}$,

$$
\begin{gathered}
T P e_{x}=\left(\left(R-R_{c r}\right)+R_{c r} \sin \psi\right) \sin (\xi+\zeta) \\
T P e_{y}=\left(\left(R-R_{c r}\right)+R_{c r} \sin \psi\right) \cos (\xi+\zeta)(8) \\
T P e_{z}=-R_{c r} \cos \psi
\end{gathered}
$$




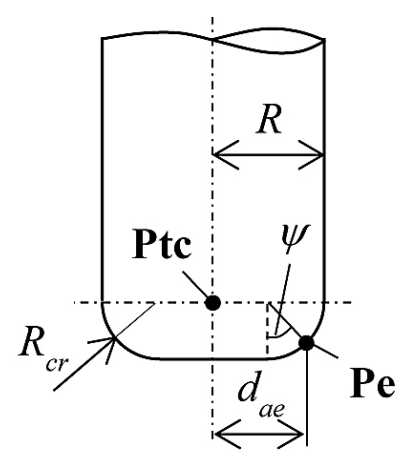

(a) Typical tool profile

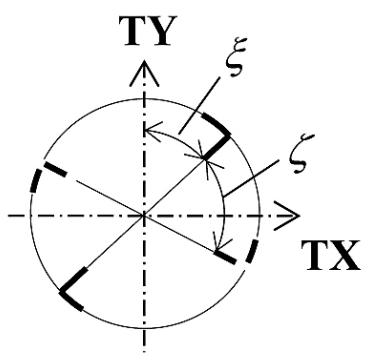

(b) Cutting edges' position in TX - TY plane

Fig. 4. Typical tool profile and cutting edges' position

and when $d_{a e}<R-R_{c r}$,

$$
\begin{gathered}
T P e_{x}=d_{a e} \sin (\xi+\zeta) \\
T P e_{y}=d_{a e} \cos (\xi+\zeta) \\
T P e_{z}=-R_{c r}
\end{gathered}
$$

It should be careful that clockwise rotation is the positive rotational direction of $\xi$ and $\zeta$. Common end mills have a number of cutting edges, so that $\xi$ depends on the number of cutting edges $n_{c e}$. In the case that the angular position of a cutting edge overlaps $\mathbf{T X}$ axis, $\xi$ can be derived as a simple form:

$$
\xi=(m-1) \frac{2 \pi}{n_{c e}}\left(1 \leq m \leq n_{c e}\right)
$$

where: $m$ is an assigned number of a cutting edge.

From the above explanation, a cutting point on a cutting edge Pe can be calculated through the following formula:

$$
\mathbf{P e}=\mathbf{R}_{\mathbf{T}} \mathbf{T P e}+\mathbf{P t c}
$$

where: $\mathbf{R}_{\mathbf{T}}$ is coordinate transformation matrix, providing a transformation from $\mathrm{T}$ to O coordinate system; moreover, Ptc is an instantaneous tool center shown in Figure 4 (a), depending on a tool path up until a present position.

The geometrical description explained in this section assumes a filleted end mill without a helix angle of cutting edges, while it can effortlessly apply to the common, commercial ones through dividing a tool into small disk elements.

\section{Tool condition parameters}

This section theoretically provides tool condition parameters favorable for smart tool management. Especially, we focus on the parameters contributable for predicting tool life and wear. They are closely related to machining conditions. Thus, the decision-making step determining them is indispensable to achieve suitable machining conditions. The some theoretical procedures will be given below with the aim of optimal parameters' selection in filleted end milling. Note that the following calculations are unalterably given for one cutting edge during one rotation of a tool.

\section{Maximum frictional distance}

Frictional distance is a critical factor to predict tool wear at the cutting edge. It also has an influence on a machined surface feature. The following formula can provide the maximum length:

$$
s l_{f d}=\left(R_{c r} \sin \alpha_{f d}+R_{b}\right) \beta_{f d}
$$

In the above formula, $\alpha_{f d}$ is obtained from an arccosine calculation for a scalar product of $\mathbf{u}_{\text {scp }}$ and -TZ. Here, a directional vector $\mathbf{u}_{\text {scp }}$ can be calculated as follows: a bull nose shape of filleted end mill is modeled as a torus created by a rotation of true circle about $\mathbf{T Z}$ axis. The circle's radius is identical to a corner radius of cutting edge, and a torus's section can be always set by rotating the true circle. In the filleted end milling, a single contact point between a workpiece's surface and the true circle inevitably arises at one moment during the rotation of cutting edge. Here, we consider the true circle's center at one moment when the single contact point arises. Then, a vector product can be calculated from a tangent vector to torus's major circle and a workpiece's surface normal. After that, $\mathbf{u}_{\text {scp }}$ is obtained as a vector product of the tangent vector to torus's major circle and the vector calculated previously. These procedures can be effortlessly implemented using the computational 
algorithm proposed in our previous study [29]. Moreover, a rotational angle around $\mathbf{T Z}$ axis $\beta_{f d}$ is utilized as the one between $\mathbf{T X}-\mathbf{T Z}$ plane and a center of torus section having a contact point with a workpiece's surface. The angles $\alpha_{f d}$ and $\beta_{f d}$ can be given in the calculation process of computational algorithm.

\section{Maximum contact arc length}

Contact arc length is defined based on a cutting-related part in a cutting edge, so that it is also an important factor in considering tool surface damage and a machined surface feature. The maximum length can be expressed:

$$
s l_{c a l}=R_{c r}\left(\alpha_{c a l}+\alpha_{h f}\right)
$$

where: $\alpha_{c a l}$ is easily identified from an angle calculation using $R_{c r}$ and the depth of cut $a p$ which means a distance between a designed surface and workpiece's surface principally.

In contrast, $\alpha_{h f}$ can be computed from an angle calculation based on a feed mark's height $h_{f}$. Here, $h_{f}$ can be mathematically expressed in the same manner to path interval determination in ball end milling. The angle $\alpha_{h f}$ is also obtainable in the calculation process of computational algorithm.

\section{Maximum cutting speed}

It is well known that cutting speed has actual impact on both tool surface damage and cutting force. The following expression can be made to estimate the maximum cutting speed:

$v_{c s}=2 \pi\left(R_{b}+R_{c r} \cos \left(\frac{\pi}{2}-\left(\theta+\alpha_{c s}\right)\right)\right) \frac{s}{1000}(14)$

where: $s$ is a spindle speed in cutting process, and $\alpha_{C S}$ is easily identified using the depth of cut $a p$.

In the calculation process of computational algorithm, the angle $\alpha_{c S}$ is available under any conditions.

\section{Average uncut chip thickness}

Uncut chip thickness is one of the vital factors having great impact on cutting force. It is undoubted that uncut chip thickness affects tool surface damage in practice since cutting force directly depends on material removal rate in machining process. The following formula enables us to calculate the average value:

$$
t_{u c t}=f_{t} \sin \beta_{u c t} \sin \alpha_{u c t}
$$

where: $\alpha_{\text {uct }}$ indicates an angular parameter for determining an arbitrary position on a cutting edge. In addition, $\beta_{\text {uct }}$ designates an angle between $\mathbf{T X}$ axis and $\mathbf{G Y}$ - GZ plane, and the rotation is about $\mathbf{T Z}$ axis.

The above formula can estimate an uncut chip thickness at any position on a cutting edge. As a brief estimation, an average uncut chip thickness $t_{a v e}$ was computed in a cutting edge's rotational position where the instantaneous cutting load and area were the largest in both measures, and three angles of $\alpha_{\text {uct }}$ were considered for the calculation. The first angle was obtained based on the depth of cut $a p$. The second angle was set to $5 \mathrm{deg}$. a priori. Finally, their median was also set to the third angle, and the average calculation was conducted with $t_{\text {uct }}$ obtained using these angles.

\section{DEMONSTRATIONS AND DISCUSSION}

Demonstrations of the tool condition parameters will be provided in this section. The demonstrations were conducted under the three conditions with a variation of tool rotational speed and feed rate. They were also performed under the condition that a cutting area entirely exists in front of the cutting-related part of filleted end mill. Moreover, the influence of tool orientation on each parameter was investigated in filleted end milling. The discussion will also be made with visualizing characteristics of these parameters. Three machining conditions used in the demonstration are shown in Table 1. The condition 1 was set with reference to recommended conditions listed in several tool catalogs. The tool rotational speed $s$ in condition 2 was twice as high as that in condition 1 . The feed rate $f$ in condition 3 was two times faster than that of condition 1. The tool diameter of end mill was set on the basis of a commonlyused size, and the tool had two straight cutting edges. The unit of angle was set as deg. to aid an intuitive understanding.

The influence of tool orientation on $s l_{f d}$ is shown in Figure 5. The same results were obtained under the all conditions. These results means that $s l_{f d}$ depended strictly on tool posture in filleted end milling. 
Table 1. The machining conditions in filleted end milling

\begin{tabular}{|l|c|c|c|}
\hline \multicolumn{1}{|c|}{ Specification } & Condition 1 & Condition 2 & Condition 3 \\
\hline Tool radius, $R[\mathrm{~mm}]$ & 6.0 & 6.0 & 6.0 \\
\hline Tool tip, $R_{c r}[\mathrm{~mm}]$ & 4.0 & 4.0 & 4.0 \\
\hline The number of tooth, $n_{c e}$ & 2 & 2 & 2 \\
\hline Depth of cut, $a p[\mathrm{~mm}]$ & 0.2 & 0.2 & 0.2 \\
\hline Tool rotational speed, $s\left[\mathrm{~min}^{-1}\right]$ & 1400 & 2800 & 1400 \\
\hline Feed rafte, $f[\mathrm{~mm} / \mathrm{min}]$ & 260 & 260 & 520 \\
\hline
\end{tabular}

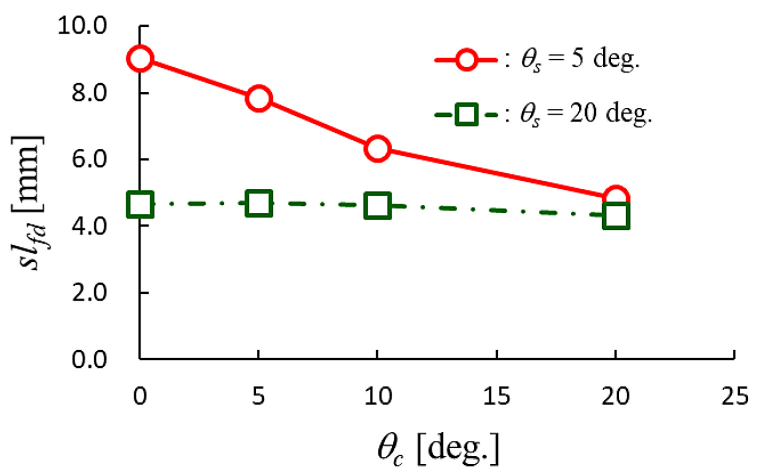

Fig. 5. Influence of $\theta_{s}$ and $\theta_{c}$ on $s l_{f d}$ under the all conditions

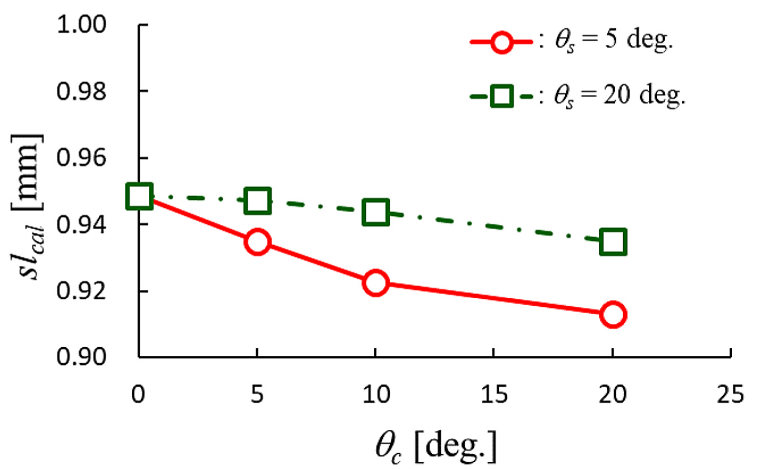

Fig. 6. Influence of $\theta_{s}$ and $\theta_{c}$ on $s l_{c a l}$ under the condition 1

In Figure 5, each curve for respective $\theta_{S}$ in graph legends represented decreasing tendency with increasing $\theta_{c}$ in any case and seemingly straight decline. Moreover, the differences between each curve became gradually smaller with increasing $\theta_{c}$. The results denoted that the values of $s l_{f d}$ appeared to converge towards a certain value with increasing $\theta_{c}$. As an example of numerical results, when $\theta_{c}=20$ deg., the values of $s l_{f d}$ were $4.82 \mathrm{~mm}$ for $\theta_{s}$ with $5 \mathrm{deg}$. and $4.32 \mathrm{~mm}$ for $\theta_{S}$ with $20 \mathrm{deg}$. From the numerical results, the values of $s l_{f d}$ are likely to become convergent in the case of $\theta_{c}$ with more than $20 \mathrm{deg}$.

The influence of tool orientation on $s l_{c a l}$ is given in Figures 6, 7 and 8. There were differences

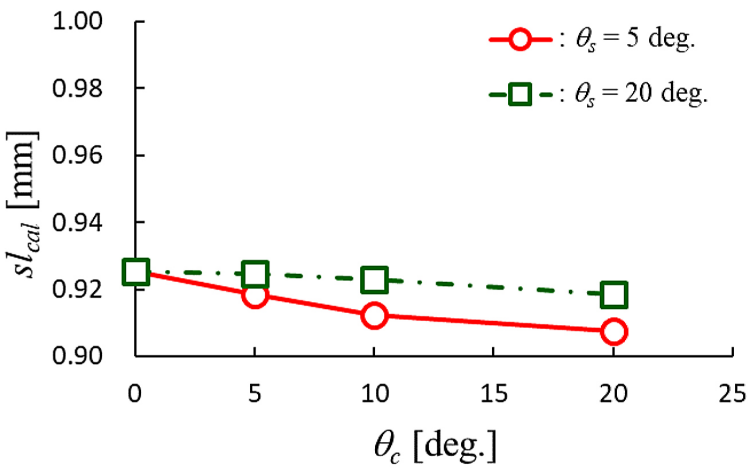

Fig. 7. Influence of $\theta_{s}$ and $\theta_{c}$ on $s l_{c a l}$ under the condition 2

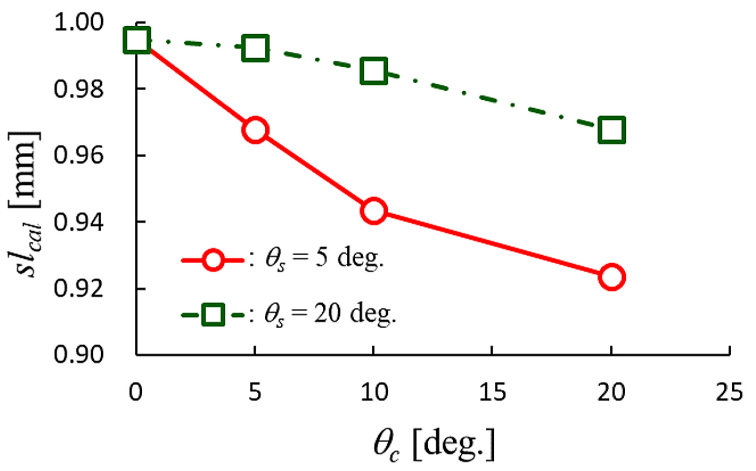

Fig. 8. Influence of $\theta_{S}$ and $\theta_{c}$ on $s l_{c a l_{1}}$ under the condition 3

between each condition since $s l_{c a l}$ depended essentially on a path interval along the feed direction of a tool. In these figures, each curve for respective $\theta_{S}$ in graph legends represented decreasing tendency with increasing $\theta_{c}$ in any case. The differences between each curve became larger with increasing $\theta_{c}$, whereas it could be presumed that there existed the limit of $s l_{c a l}$ in the case of $\theta_{c}$ having an angle more than $20 \mathrm{deg}$. Although a certain line graph in condition 3 was seemingly observed as a precipitous decrease, the all results indicated that contact arc length was numerically unaltered in any case. In the pre-determined condition of $z$, the values of $s l_{c a l}$ were 0.95 $\mathrm{mm}$ in any case when $\theta_{c}=0 \mathrm{deg}$. in condition 1 . The values were $0.93 \mathrm{~mm}$ in any case when $\theta_{c}=0$ 
deg. in condition 2 . They were $0.99 \mathrm{~mm}$ in any case when $\theta_{c}=0$ deg. in condition 3. In addition, when $\theta_{c}=20$ deg., the values of $s l_{c a l}$ were $0.91 \mathrm{~mm}$ for $\theta_{S}$ with $5 \mathrm{deg}$. and $0.93 \mathrm{~mm}$ for $\theta_{S}$ with $20 \mathrm{deg}$. in condition 1 . The values were $0.91 \mathrm{~mm}$ for $\theta_{S}$ with 5 deg. and $0.92 \mathrm{~mm}$ for $\theta_{s}$ with $20 \mathrm{deg}$. in condition 2 . They were $0.92 \mathrm{~mm}$ for $\theta_{S}$ with $5 \mathrm{deg}$. and $0.97 \mathrm{~mm}$ for $\theta_{S}$ with $20 \mathrm{deg}$. in condition 2 . From the numerical values, there were noticeable differences under the case that $\theta_{S}$ and $\theta_{c}$ equal $20 \mathrm{deg}$.

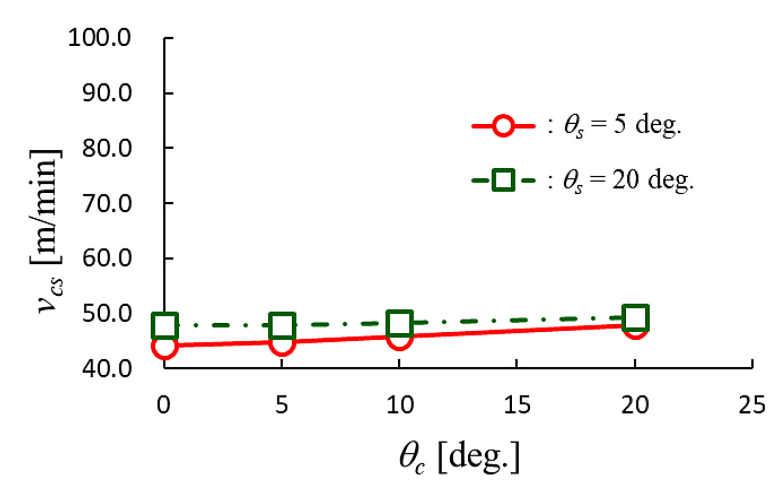

Fig. 9. Influence of $\theta_{S}$ and $\theta_{c}$ on $v_{C S}$ under the conditions 1 and 3

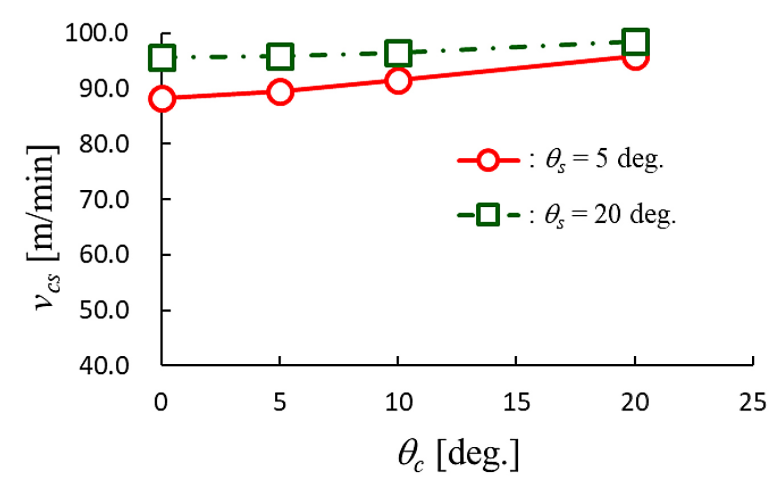

Fig. 10. Influence of $\theta_{S}$ and $\theta_{C}$ on $v_{C S}$ under the condition 2

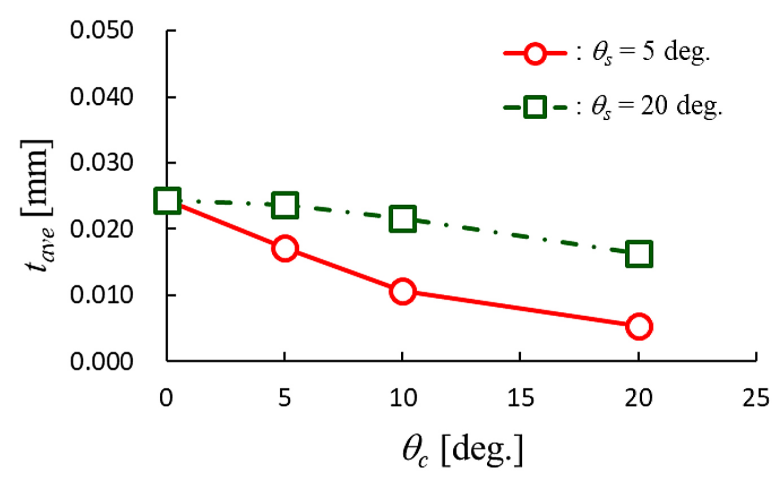

Fig. 11. Influence of $\theta_{s}$ and $\theta_{c}$ on $t_{\text {ave }}$ under the condition 1
The influence of tool orientation on $V_{C S}$ is represented in Figures 9 and 10. The same results were observed under the conditions 1 and 3 , whereas the different result was obtained under the condition 2. In these figures, each curve for respective $\theta_{S}$ in graph legends showed slowly increasing tendency with increasing $\theta_{c}$ in any case. The increasing tendency considerably diminished with increasing $\theta_{s}$. Moreover, the differences between each curve became smaller with increasing $\theta_{c}$. In conditions 1 and 3 , when $\theta_{S}=20 \mathrm{deg}$. and $\theta_{c}=5$ deg., the value of $v_{C S}$ was $47.92 \mathrm{~m} /$ min. Likewise, when $\theta_{S}=5 \mathrm{deg}$. and $\theta_{C}=20 \mathrm{deg}$., the value of $v_{C S}$ was $47.92 \mathrm{~m} / \mathrm{min}$. In condition 2 , when $\theta_{S}=20 \mathrm{deg}$. and $\theta_{C}=5 \mathrm{deg}$., the value of $v_{C S}$ was $95.84 \mathrm{~m} / \mathrm{min}$. Similarly, when $\theta_{S}=5 \mathrm{deg}$. and $\theta_{C}=20 \mathrm{deg}$., the value of $v_{C S}$ was $95.84 \mathrm{~m} /$ min. In the four machining states, the value of $\theta$ was $20.52 \mathrm{deg}$. It was obvious from the results that the value of $v_{C S}$ was completely identical under the same $\theta$ and depended strongly on $s$.

The influence of tool orientation on $t_{a v e}$ is displayed in Figures 11, 12, and 13. There were differences between each condition since $t_{a v e}$ depended

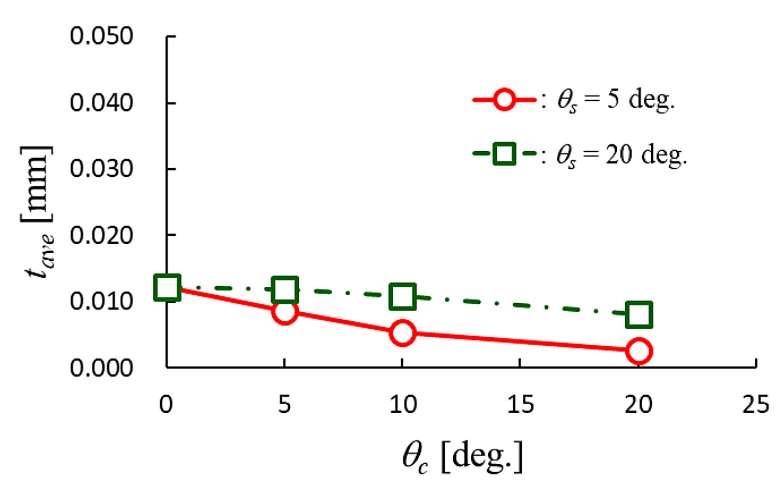

Fig. 12. Influence of $\theta_{S}$ and $\theta_{c}$ on $t_{\text {ave }}$ under the condition 2

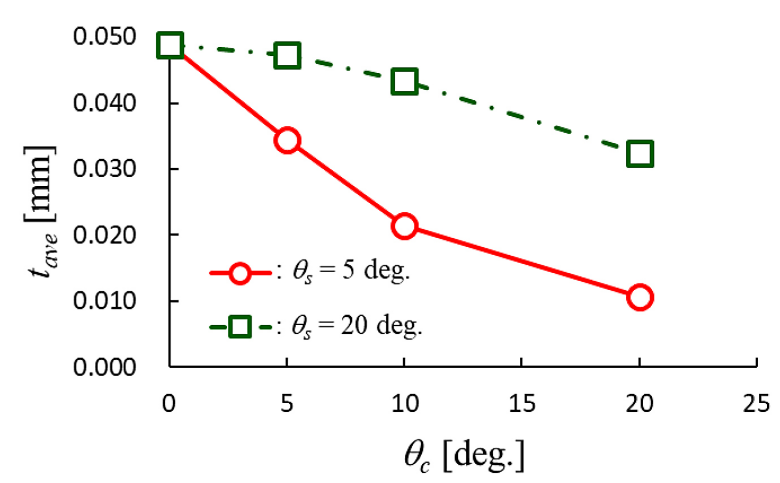

Fig. 13. Influence of $\theta_{S}$ and $\theta_{c}$ on $t_{\text {ave }}$ under the condition 3 
essentially on a path interval along the feed direction of a tool. The feature was very similar to that of $s l-$ cal. In these figures, each curve for respective $\theta_{S}$ in graph legends indicated decreasing tendency with increasing $\theta_{c}$ in any case. The differences between each curve became larger with increasing $\theta_{c}$, whereas it can be presumed that there existed the limit of $t_{\text {ave }}$ in the case of $\theta_{c}$ having an angle more than 20 deg. The results implied that an instantaneous cutting force acting on a cutting edge decreased with increasing $\theta$. With reference to the variation of $t_{\text {ave, }}$ the decreasing rate would be especially prominent in the small value of $\theta_{S}$.

\section{CONCLUSION}

This study dealt with theoretical investigations regarding tool condition parameters. The computational analyses were demonstrated to determine machining conditions after the geometrical modeling was provided to express multi-axis filleted end milling. The demonstrations with three machining conditions were made to compare the results under the conditions with some variations of $\theta_{s}, \theta_{c}, s$, and $f$. As a result, some characteristics of tool condition parameters were revealed from the visual evidences and discussion. One of the characteristics was that $s l_{f d}$ depended strictly on tool posture in filleted end milling. Moreover, $s l$ cal depended essentially on a path interval along the feed direction of a tool. The results obtained from the demonstrations also represented that $t_{a v e}$ could be indirectly predictable from $s l_{c a l}$.

As a future work, the further detailed analysis will be conducted in a wide variety of conditions, and the experimental verification will be made to evaluate validity and applicability of tool condition parameters proposed in this study.

\section{Acknowledgments}

The authors would like to thank the financial support provided by OSG Fund and the research grant from Faculty of Science and Technology, Seikei University.

\section{REFERENCES}

1. Dantas T.E.T., De-Souza E.D., Destro I.R., Hammes G., Rodriguez C.M.T., Soares S.R. How the combination of Circular Economy and Industry
4.0 can contribute towards achieving the Sustainable Development Goals. Sustainable Production and Consumption. 2021;26:213-227.

2. Bhatt Y., Ghuman K., Dhir A. Sustainable manufacturing. Bibliometrics and content analysis. Journal of Cleaner Production. 2020;260:120988.

3. Kishawy H.A., Hegab H., Saad E. Design for Sustainable Manufacturing: Approach, Implementation, and Assessment. Sustainability. 2018;10:3604.

4. Ahmad S., Wong K.Y., Tseng M.L., Wong W.P. Sustainable product design and development: A review of tools, applications and research prospects. Resources, Conservation and Recycling. 2018;132:49-61.

5. Castro G.D.R., Fernández M.C.G., Colsa Á.U. Unleashing the convergence amid digitalization and sustainability towards pursuing the Sustainable Development Goals (SDGs): A holistic review. Journal of Cleaner Production. 2021;280:122204.

6. Zarte M., Pechmann A., Nunes I.L. Decision support systems for sustainable manufacturing surrounding the product and production life cycle $-\mathrm{A}$ literature review. Journal of Cleaner Production. 2019;219:336-349.

7. Salem A., Hegab H., Kishawy H.A. An integrated approach for sustainable machining processes: Assessment, performance analysis, and optimization. Sustainable Production and Consumption. 2021;25:450-470.

8. Equeter L., Ducobu F., Dehombreux P. Cutting Tools Replacement: Toward a Holistic Framework. IFAC-PapersOnLine. 2020;53:227-232.

9. Xie Y., Lian K., Liu Q., Zhang C., Liu H. Digital twin for cutting tool: Modeling, application and service strategy. Journal of Manufacturing Systems. 2021;58:305-312.

10. Sun H., Liu Y., Pan J., Zhang J., Ji W. Enhancing cutting tool sustainability based on remaining useful life prediction. Journal of Cleaner Production. 2020;244:118794.

11. Ambhore N., Kamble D., Chinchanikar S., Wayal V. Tool Condition Monitoring System: A Review, Materials Today. Proceedings. 2015;2:3419-3428.

12. Nath C. Integrated Tool Condition Monitoring Systems and Their Applications: A Comprehensive Review. Procedia Manufacturing. 2020;48:852-863.

13. Kuntoğlu M., Aslan A., Pimenov D.Y., Usca Ü.A., Salur E., Gupta M.K., Mikolajczyk T., Giasin K., Kapłonek W., Sharma S. A Review of Indirect Tool Condition Monitoring Systems and DecisionMaking Methods in Turning: Critical Analysis and Trends. Sensors. 2021;21:108.

14. Siddhpura A., Paurobally R., A review of flank wear prediction methods for tool condition monitoring in a turning process. Int J Adv Manuf Technol. 2013;65:371-393. 
15. Serin G., Sener B., Ozbayoglu A.M., Unver H.O. Review of tool condition monitoring in machining and opportunities for deep learning. Int $\mathrm{J} \mathrm{Adv}$ Manuf Technol. 2020;109:953-974.

16. Patange A.D., Jegadeeshwaran R. Review on tool condition classification in milling: A machine learning approach. Materials Today: Proceedings; 2021.

17. Jeon B., Yoon J.S., Um J., Suh S.H. The architecture development of Industry 4.0 compliant smart machine tool system (SMTS). J Intell Manuf. 2020;31:1837-1859.

18. Mohanraj T., Shankar S., Rajasekar R., Sakthivel N.R., Pramanik A. Tool condition monitoring techniques in milling process - a review. Journal of Materials Research and Technology. 2020;9:1032-1042.

19. Xu X. Machine Tool 4.0 for the new era of manufacturing, Int J Adv Manuf Technol. 2017;92:1893-1900.

20. Altintas Y., Brecher C., Weck M., Witt S. Virtual machine tool, CIRP Annals - Manufacturing Technology. 2005;54:651-704.

21. Takeuchi Y. CAM for Multi-Axis Control and Multi-Tasking Machining, Trans. JSME Series C. 2011;77:3544-3551. (in Japanese)

22. Konobrytskyi D., Hossain M.M., Tucker T.M., Tarbutton J.A., Kurfess T.R. 5-Axis tool path planning based on highly parallel discrete volumetric geometry representation: Part I contact point generation. Computer-Aided Design and Applications. 2018; 15:76-89.

23. Lasemi A., Xue D., Gu P. Recent development in CNC machining of freeform surfaces: a state-of-theart review. Comput. Aided Des. 2010;42:641-654.

24. Bo P., Bartoň M., Plakhotnik D., Pottmann H. Towards efficient 5-axis flank CNC machining of free-form surfaces via fitting envelopes of surfaces of revolution. Comput. Aided Des. 2016;79:1-11.

25. Harik R.F., Gong H., Bernard A. 5-axis flank milling: A state-of-the-art review. Comput. Aided Des. 2013;45:796-808.

26. Sekine T., Obikawa T. Normal-Unit-Vector-Based Tool Path Generation Using a Modified Local Interpolation for Ball-End Milling, Journal of Advanced Mechanical Design, Systems, and Manufacturing. 2010;4:1246-1260.

27. Mladenovic G.M., Tanovic L.M., Ehmann K.F. Tool path generation for milling of free form surfaces with feedrate scheduling. FME Transactions. 2015;43:9-15.
28. Sekine T., Obikawa T. Novel path interval determination in 5-axis flat end milling. Applied Mathematical Modelling. 2015;39:3459-3480.

29. Sekine T. A Computational Algorithm for Path Interval Determination in Multi-Axis Filleted End Milling. Adv. Sci. Technol. Res. J. 2020;14:198-205.

30. Sekine T., Kameya K. Remarkable characteristics of a novel path interval determination in filleted end milling. Journal Européen des Systèmes Automatisés. 2021;54. (in press)

31. Lazoglu I., Khavidaki S.E.L., Mamedov A., Erdim H. Process Optimization via Feedrate Scheduling in Milling. In: The International Academy for Production Engineering, Laperrière L., Reinhart G. (eds) CIRP Encyclopedia of Production Engineering. Springer, Berlin, Heidelberg; 2014.

32. Habibi M., Kilic Z.M., Altintas Y. Minimizing Flute Engagement to Adjust Tool Orientation for Reducing Surface Errors in Five-Axis Ball End Milling Operations, ASME. J. Manuf. Sci. Eng. 2021;143:021009.

33. Zhang X., Zhang J., Zheng X., Pang B., Zhao W. Tool orientation optimization of 5-axis ball-end milling based on an accurate cutter/workpiece engagement model. CIRP Journal of Manufacturing Science and Technology. 2017;19:106-116.

34. Lotfi S., Rami B., Maher B., Gilles D., Wassila B. An approach to modeling the chip thickness and cutter workpiece engagement region in 3 and 5 axis ball end milling. J. Manuf. Process. 2018;34:7-17.

35. Masmiati N., Sarhan A.A.D. Optimizing cutting parameters in inclined end milling for minimum surface residual stress - Taguchi approach. Measurement. 2015;60:267-275.

36. Wojciechowski S., Maruda R.W., Nieslony P., Krolczyk G.M. Investigation on the edge forces in ball end milling of inclined surfaces. International Journal of Mechanical Sciences. 2016;119:360-369.

37. Budak E., Ozlu E. Development of a thermomechanical cutting process model for machining process simulations. CIRP Annals. 2008;57:97-100.

38. Soori M., Arezoo B. Virtual Machining Systems for CNC Milling and Turning Machine Tools: A Review, International Journal of Engineering and Future Technology, 2021;18:56-104.

39. Davim J.P. Machining of Complex Sculptured Surfaces, Springer; 2012. 